\title{
SNX3 controls Wingless/Wnt secretion through regulating retromer-dependent recycling of Wntless
}

\author{
Peng Zhang ${ }^{1, *}$, Yihui $\mathrm{Wu}^{1, *}$, Tatyana Y Belenkaya ${ }^{2}$, Xinhua $\operatorname{Lin}^{1,2}$ \\ ${ }^{I}$ State Key Laboratory of Biomembrane and Membrane Biotechnology, and Key Laboratory of Stem Cell and Developmental \\ Biology, Institute of Zoology, Chinese Academy of Sciences, Beijing 100101, China; ${ }^{2}$ Division of Developmental Biology, \\ Cincinnati Children's Hospital Medical Center, Cincinnati, OH 45229, USA
}

\begin{abstract}
Drosophila Wingless (Wg) acts as a morphogen during development. Wg secretion is controlled by a sevenpass transmembrane cargo Wntless (Wls). We have recently identified retromer as a key regulator involved in Wls trafficking. As sorting nexin (SNX) molecules are essential components of the retromer complex, we hypothesized that specific $\operatorname{SNX}(s)$ is required for retromer-mediated Wnt secretion. Here, we generated Drosophila mutants for all of the eight snx members, and identified Drosophila SNX3 (DSNX3) as an essential molecule required for Wg secretion. We show that $\mathrm{Wg}$ secretion and its signaling activity are defective in Dsnx3 mutant clones in wing discs. Wg levels in the culture medium of Dsnx3-depleted S2 cells are also markedly reduced. Importantly, Wls levels are strikingly reduced in Dsnx 3 mutant cells, and overexpression of Wls can rescue the Wg secretion defect observed in Dsnx3 mutant cells. Moreover, DSNX3 can interact with the retromer component Vps35, and co-localize with Vps35 in early endosomes. These data indicate that DSNX3 regulates $\mathrm{Wg}$ secretion via retromer-dependent Wls recycling. In contrast, we found that Wg secretion is not defective in cells mutant for Drosophila snx1 and snx6, two components of the classical retromer complex. Ectopic expression of DSNX1 or DSNX6 fails to rescue the Wg secretion defect in Dsnx3 mutant wing discs and in Dsnx3 dsRNA-treated S2 cells. These data demonstrate the specificity of the DSNX3retromer complex in Wls recycling. Together, our findings suggest that DSNX3 acts as a cargo-specific component of retromer, which is required for endocytic recycling of Wls and $\mathrm{Wg} / \mathrm{Wnt}$ secretion.
\end{abstract}

Keywords: Drosophila SNX3 (DSNX3); Wnt; Wingless (Wg); Wntless (Wls); retromer; signaling Cell Research (2011) 21:1677-1690. doi:10.1038/cr.2011.167; published online 1 November 2011

\section{Introduction}

Wnt proteins are evolutionarily conserved glycoproteins that play profound roles in a variety of developmental processes as well as in adult homeostasis [1]. Abnormal Wnt signaling is associated with many human diseases, ranging from cancers to degenerative diseases $[2,3]$. Drosophila Wingless (wg) encodes the homolog of vertebrate Wnt-1 $[4,5]$. Wg acts as a short-range organizer and a long-range morphogen in a variety of developmental contexts $[6,7]$. How Wnt processing

*These two authors contributed equally to this work.

Correspondence: Xinhua Lin

Tel/Fax: +86-10-64807970

E-mail: xinhua.lin@ioz.ac.cn; xinhua.lin@cchmc.org

Received 21 July 2011; revised 19 September 2011; accepted 26 September 2011; published online 1 November 2011 and secretion are regulated is an essential issue in the Wnt field, yet the underlying mechanisms are not fully understood [8].

Genetic screens in Drosophila identified Wntless (Wls, also known as Evenness Interrupted (Evi) or Sprinter (Srt)) as an essential regulator for $\mathrm{Wg} / \mathrm{Wnt}$ secretion [9-11]. Wls is a seven-pass transmembrane protein that is localized in the Golgi apparatus, at the cell surface and in early endosomes [9, 12-14]. The function of Wls in Wg/ Wnt secretion is conserved from Drosophila, C. elegans to vertebrates. In Drosophila wls mutants, $\mathrm{Wg}$ is not secreted and is accumulated inside Wg-producing cells [9-11]. Recent studies have also shown that Gpr177, the mouse homolog of Drosophila wls, is essential for Wnt secretion during embryonic development $[15,16]$.

We and others have recently identified retromer as an essential regulator for $\mathrm{Wg} / \mathrm{Wnt}$ secretion in $\mathrm{Wg} / \mathrm{Wnt}$ producing cells [12-14, 17-20]. Our studies as well as 
others demonstrate that Wls endocytosis is a clathrinmediated process in which retromer plays an essential role in Wls retrograde recycling from early endosomes to the trans-Golgi network (TGN) [12-14, 17, 18]. Retromer is an evolutionarily conserved multi-protein complex whose function is to sort cargo from early endosomes back to TGN [21-23]. In yeast, the retromer complex consists of two independently assembled subcomplexes: the cargo selective VPS subcomplex, consisting of Vps35p, Vps26p, and Vps29p and the membrane-binding dimer formed by Vps5p and Vps17p. Vps5p and Vps17p are sorting nexins (SNXs), a protein family containing a phosphoinositide-binding PX (phox homology) domain that binds phosphatidylinositol 3-phosphate (PI3P) and other phosphoinositides [24-26]. Some of the SNXs also have a BAR (Bin/amphiphysin/Rvs) domain, which is a dimerization and membrane-binding module capable of sensing membrane curvatures [24]. In mammals, SNX1 and SNX2 are the orthologs of yeast Vps5p [27, 28], while SNX5 and SNX6 appear to be the functional orthologs of yeast Vps17p [29]. SNX1/SNX2 (SNX1/2) and SNX5/SNX6 (SNX5/6) all contain BAR domains [24]. The current model for the classic retromer complex is that one chain of SNX1 or SNX2 and one chain of SNX5 or SNX6 interact with the VPS subcomplex to form the functional retromer complex required for retrograde transport of cargo proteins such as the phagocytic receptor CED-1 in C. elegans [30], and the cation-independent mannose 6-phosphate receptor (CIMPR) in mammals [23, 31-35]. Recent studies further demonstrated that SNX5/6 can interact with the $\mathrm{p} 150^{\text {Glued }}$ subunit of the dynein/dynactin motor complex and that such interaction is important to drive the formation and movement of tubular retrograde intermediates [34, 35]. In addition to the SNX-BAR proteins, previous studies have shown that yeast Grd19p, a SNX3 homolog lacking the BAR domain, can also function in retromer-dependent endosome-to-Golgi retrieval of the iron transporter Fet3p-Ftr1p, a cargo protein [36-38]. Very recently, SNX27, another non-BAR SNX molecule, was shown to act as a retromer component for recycling the $\beta 2$ adrenergic receptor to the plasma membrane [39]. Currently, it is unknown whether different SNX molecules can interact with the VPS subcomplex to form a specific retromer subcomplex required for cargo selection and retrograde transport.

To identify specific SNX molecules involved in retromer-mediated $\mathrm{Wg}$ secretion, we generated mutants of individual Drosophila snx genes and analyzed their roles in $\mathrm{Wg}$ secretion and signaling. To our surprise, we found that Drosophila SNX3 (DSNX3), but not the SNX-BAR proteins SNX1/2 and SNX5/6, is required for
$\mathrm{Wg}$ secretion. We show that $\mathrm{Wg}$ secretion is defective in the absence of DSNX3 activity. Moreover, DSNX3 interacts with Vps35 and Wls, and regulates Wls stability. Our data argue that DSNX3 interacts with the VPS subcomplex to form a retromer subcomplex required for endocytic recycling of Wls.

\section{Results}

Identification of Dsnx3 as an essential candidate gene involved in $\mathrm{Wg}$ secretion

Searches of Drosophila genome databases identified eight snx genes, including CG2774, Dsnx6/CG8282, CG6359, SH3PX1/CG6757, CG5734, CG3077, Snz/ $C G 1514$ and $C G 32758$. To examine the roles of these snx genes in development, we generated null alleles of seven Drosophila snx genes by $\mathrm{P}$ element-mediated imprecise excision. Null mutant for Dsnx6 was obtained by the "Ends-out" gene targeting technique [40], as there was no available $\mathrm{P}$ element inserted near the $D \sin x 6$ gene. DSNX6 is the homolog of mammalian SNX5/6. The protein product of CG2774 is highly homologous to both yeast Vps5p and mammalian SNX1/2, and therefore we referred to CG2774 as Drosophila snxl (Dsnx1). The protein product of CG6359 is highly homologous to mammalian SNX3 or SNX12, therefore is referred to as DSNX3. Previous studies in mammalian systems have shown that SNX1/2 and SNX5/6 are the components of the classic retromer complex [27, 29, 34, 35]. Therefore, we speculated that DSNX1 and DSNX6 may be the critical SNX molecules required for retromer-mediated Wg secretion. To our surprise, Dsnx 1 null mutants were completely viable and fertile, and exhibited no detectable defects in $\mathrm{Wg}$ signaling determined by genetic mosaic analysis in wing discs (see data later). Similarly, the Dsnx6 null mutants were also completely viable and fertile, and exhibited no obvious defects (data not shown). In contrast, Dsnx3 null mutants were semilethal, and the fly wings of homozygous $D \operatorname{sn} x 3$ mutants extended from the body axis. Using the "direct mosaic system" [41], we generated Dsnx3 mutant mosaic clones in developing wings by vg Q1206-Gal4 UAS-Flp, and found obvious notching at the margin region of the wing (Supplementary information, Figure S1B and S1B'), indicating that DSNX3 is involved in Wg signaling.

In the wing disc, the $\mathrm{Wg}$ protein is produced at the dorso/ventral $(\mathrm{D} / \mathrm{V})$ boundary and forms a gradient along the $\mathrm{D} / \mathrm{V}$ axis [42] (Figure 1A). Wg induces senseless (sens) expression at a short range, whereas it activates distalless $(\mathrm{dll})$ at a long range [43-45]. We examined the requirement of DSNX3 for Wg secretion and signaling by genetic mosaic analysis in wing discs using the 

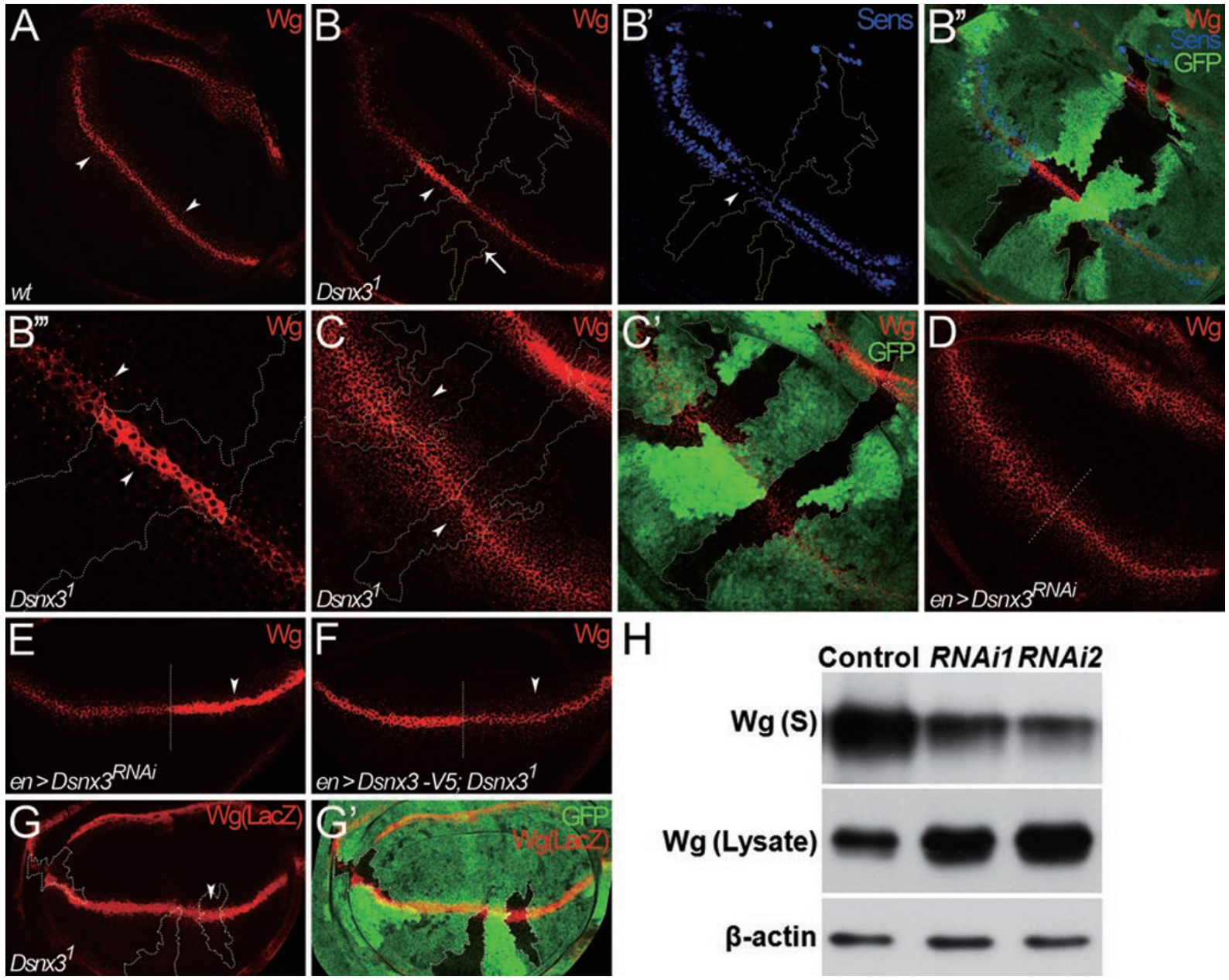

Figure 1 DSNX3 is required for Wg secretion and signaling. (A-D) Wing discs are oriented dorsal top-right, anterior topleft. (A) Wg staining in wild-type disc. Wg was detected in its producing cells along D/V boundary and outside of producing cells in punctate structures (arrowheads). (B-B") Wg and Sens staining in a wing disc bearing Dsn $\times 3^{1}$ clones. Mutant clones are outlined by the dotted lines to mark the absence of GFP (B", green). Wg was accumulated inside Dsnx3 mutant Wgproducing cells, but was not altered in a mutant clone residing outside of the Wg-producing cells marked by yellow dotted lines (arrow in B). Sens staining was dramatically reduced in Dsnx $3^{1}$ clones (arrowhead in B'). (B'"') Enlarged view of clone in $\mathbf{B}$ shows accumulated $\mathrm{Wg}$ inside Dsnx3 mutant Wg-producing cells. Also noted is the reduced number of Wg punctate structures outside Wg-producing cells in the clone regions (arrowheads in B'"). (C-C') Extracellular Wg distribution was examined in $D s n \times 3^{1}$ clones (C) marked by the dotted lines and by the absence of GFP (C', green). Extracellular Wg is reduced in the $D s n \times 3^{1}$ mutant $\mathrm{Wg}$-producing cells. (D) Extracellular $\mathrm{Wg}$ distribution in the $\mathrm{P}$ compartment of a wing disc expressing UAS-Dsnx $3^{R N A i}$ using en ${ }^{\text {Gal4 }}$. The extracellular Wg level was reduced in the $\mathrm{P}$ compartment where DSNX3 activity was eliminated by UAS-Dsnx $3^{R N A i}$. (E-G) Wing discs are oriented anterior left, dorsal up. (E) UAS-Dsnx $3^{R N A i}$ was expressed in wing discs using en $n^{\text {Gal }}$ to deplete DSNX3 activity in the $\mathrm{P}$ compartment. Anterior/posterior (A/P) boundary is shown by the dotted line. Wg accumulated in Wg-producing cells of the $\mathrm{P}$ compartment and $\mathrm{Wg}$ punctate structures in surrounding cells were almost absent in the P compartment (arrowhead). (F) In Dsnx3 homozygous mutant background, UAS-Dsnx3-V5 was expressed in the $\mathrm{P}$ compartment using $\mathrm{en}^{\text {Gal4 }}$. Wg secretion appeared to be normal in the P compartment (arrowhead), which exhibited secreted Wg punctates outside Wg-producing cells and no accumulation of Wg in Wg-producing cells. (G-G') Wg expression was monitored by $W^{l a c z}$ in $D s n \times 3^{1}$ mutant $\mathbf{W g}$-producing cells (G) marked by the dotted lines and by the absence of GFP (G', green). As shown in G (arrowhead), Wg expression was not altered. (H) The amount of Wg in the supernatant (S) was strongly reduced when $\mathrm{S} 2+\mathrm{pMK}-\mathrm{Wg}$ cells were treated with dsRNAs targeted to different regions of Dsnx3 compared to the control (GFP dsRNA). However, the amount of Wg in cell lysates was markedly increased. Both RNAi1 and RNAi2 were targeted to $D s n \times 3$ coding region. $\beta$-actin showed equal loading of lysate samples and was not altered by Dsnx3 RNAi treatment. 
FLP-FRT method [46] or by RNA interference (RNAi) to deplete Dsnx3. Wg-producing cells mutant for Dsnx3 had higher levels of $\mathrm{Wg}$ compared with surrounding wild-type cells (Figure 1B). However, Wg levels were not altered in a mutant clone residing outside of the $\mathrm{Wg}$ producing cells (marked by yellow dotted lines and an arrow in Figure 1B). Similarly, when $U A S-D \operatorname{sn} x 3^{R N A i}$ was expressed in the entire posterior $(\mathrm{P})$ compartment using $e n^{\text {Gal4 }}, \mathrm{Wg}$ protein accumulated in $\mathrm{Wg}$-producing cells and was significantly reduced in Wg-receiving cells in the P compartment (Figure 1E). Furthermore, in a $D \operatorname{sn} x 3$ homozygous mutant background, ectopic expression of DSNX3 using $e n^{\text {Gal4 }}$ can rescue the Wg secretion defect in the $\mathrm{P}$ compartment (Figure $1 \mathrm{~F}$ ). $\mathrm{Wg}$ accumulation in the mutant cells was not due to increased wg transcription, as no difference was observed in the activity of a $W g^{\text {LacZ }}$ reporter between mutant and wild-type cells (Figure $1 \mathrm{G}$ and $1 \mathrm{G}^{\prime}$ ). Furthermore, using an extracellular staining technique [47], we observed reduced levels of extracellular Wg in Dsnx3 mutant clones (Figure 1C and 1C'). Similarly, extracellular Wg levels were reduced in the $\mathrm{P}$ compartment of $\mathrm{Wg}$-producing cells and $\mathrm{Wg}$ receiving cells when $D \operatorname{sn} x 3$ activity was eliminated by $U A S-D \operatorname{sn} x 3^{R N A i}$ using en ${ }^{\text {Gal }}$ (Figure 1D). These data argue that $\mathrm{Wg}$ protein accumulated inside $\mathrm{Wg}$-producing cells in the absence of DSNX3.

We further examined the requirement of DSNX3 function for sens expression in the wing disc. Sens was reduced in Dsnx3 mutant clones straddling the $w g$ expression domain (Figure 1B'). However, Sens expression was still present in some Dsnx 3 mutant cells when they neighbored wild-type Wg-producing cells (Supplementary information, Figure S2A and S2A'), arguing that DSNX3 is not essential for $\mathrm{Wg}$ signaling per se. This result also suggests that accumulated $\mathrm{Wg}$ protein inside Dsnx3 mutant cells failed to activate $\mathrm{Wg}$ signaling. Taken together, these data suggest that loss of DSNX3 activity causes a defect in Wg secretion, subsequently leading to defects in $\mathrm{Wg}$ target gene expression.

To further confirm the requirement of DSNX3 for $\mathrm{Wg}$ secretion, we examined the effect of DSNX3 on Wg secretion in $\mathrm{S} 2 \mathrm{R}+$ cells expressing $\mathrm{Wg}$. As shown in Figure $1 \mathrm{H}, \mathrm{Wg}$ was reduced in the supernatants of cells treated with $D s n x 3$ dsRNAs against two different regions of the Dsnx 3 gene, whereas control cells treated with GFP dsRNA secreted Wg normally. Taken together, the above results strongly argue that DSNX3 activity is required for Wg secretion.

DSNX3 is required for Wg secretion in other tissues, but not essential for other signaling pathways

$\mathrm{As} \mathrm{Wg}$ is required for the development of other larval imaginal discs, we examined the role of DSNX3 in $\mathrm{Wg}$ secretion in other tissues. As shown in Figure 2, Wg was accumulated in both leg and haltere discs in the absence of DSNX3 activity (Figure 2D-2F'). Similarly, Wg was also accumulated in the $D \operatorname{sn} x 3$ mutant $\mathrm{Wg}$-producing cells of the wing hinge region (arrowhead in Figure 2D). These data suggest that DSNX3 is critical for $\mathrm{Wg}$ secretion in other tissues.

To determine the specificity of DSNX3, we examined whether DSNX3 is required for the activity of two other morphogens, Hedgehog (Hh) and Decapentaplegic (Dpp). In contrast to $\mathrm{Wg}$ secretion, we found that $\mathrm{Hh}$ secretion was normal (Figure 2A and 2A'), and that Hh-induced Patched (Ptc) expression was not altered (Figure 2B and 2B'). Similarly, Dpp signaling as determined by Sal expression was not altered (Figure $2 \mathrm{C}$ and $2 \mathrm{C}^{\prime}$ ). As $w g$ transcription in wing discs is controlled by Notch signaling [48] and was not affected in Dsnx3 mutant cells (Figure $1 \mathrm{G}$ and $1 \mathrm{G}^{\prime}$ ), we suggest that DSNX3 activity is also not required for Notch signaling. Taken together, our data suggest that DSNX3 is specifically required for $\mathrm{Wg}$ secretion.

\section{Wls stability is regulated by DSNX3}

As Wls is required for Wg secretion [9-11], one possibility is that DSNX3 may control Wg secretion by regulating Wls activity. We first examined whether DSNX3 interacts with Wls in vivo. When Dsnx3-GFP and wls$V 5$ were expressed in wing discs, Wls was present in vesicle-like punctate structures, and the majority of Wls co-localized with DSNX3 (Figure 3A-3A"). The majority of DSNX3 also co-localized with the early endosome marker GFP-2×FYVE [49] (Figure 3B-3B"). Previous studies have shown that Wls is located in early endosomes $[12,14]$. The above data suggest that DSNX3 may interact with Wls in early endosomes. Consistent with this view, DSNX3 co-localized with human Wls (hWls) and the early endosome marker early endosomal antigen 1 (EEA1) [50] when DSNX3 and hWls were coexpressed in HeLa cells (Figure 3C-3C'").

Next, we intended to determine whether Wls trafficking was regulated by DSNX3. In the wing disc, Wls is ubiquitously expressed; however, increased Wls staining is observed in a stripe at the $\mathrm{D} / \mathrm{V}$ boundary (Figure 3D), a region known to secrete and receive high levels of $\mathrm{Wg}$ [14]. Endogenous Wls levels were strikingly reduced in Dsnx 3 mutant clones (Figure 3E and 3E'). Similarly, we expressed $U A S-D s n x 3^{R N A i}$ in the posterior (P) compartment using $e n^{\text {Gal4 }}$ or $h h^{\text {Gal4 }}$, and found that Wls staining was lost in the Wg-secreting cells (arrowhead in Figure $3 \mathrm{~F}$ ) and markedly reduced in the non-Wg-secreting cells (Supplementary information, Figure S3A-S3A"). Furthermore, in flies carrying the $h s-w l s-V 5$ construct, ectopically expressed Wls levels were strikingly reduced 

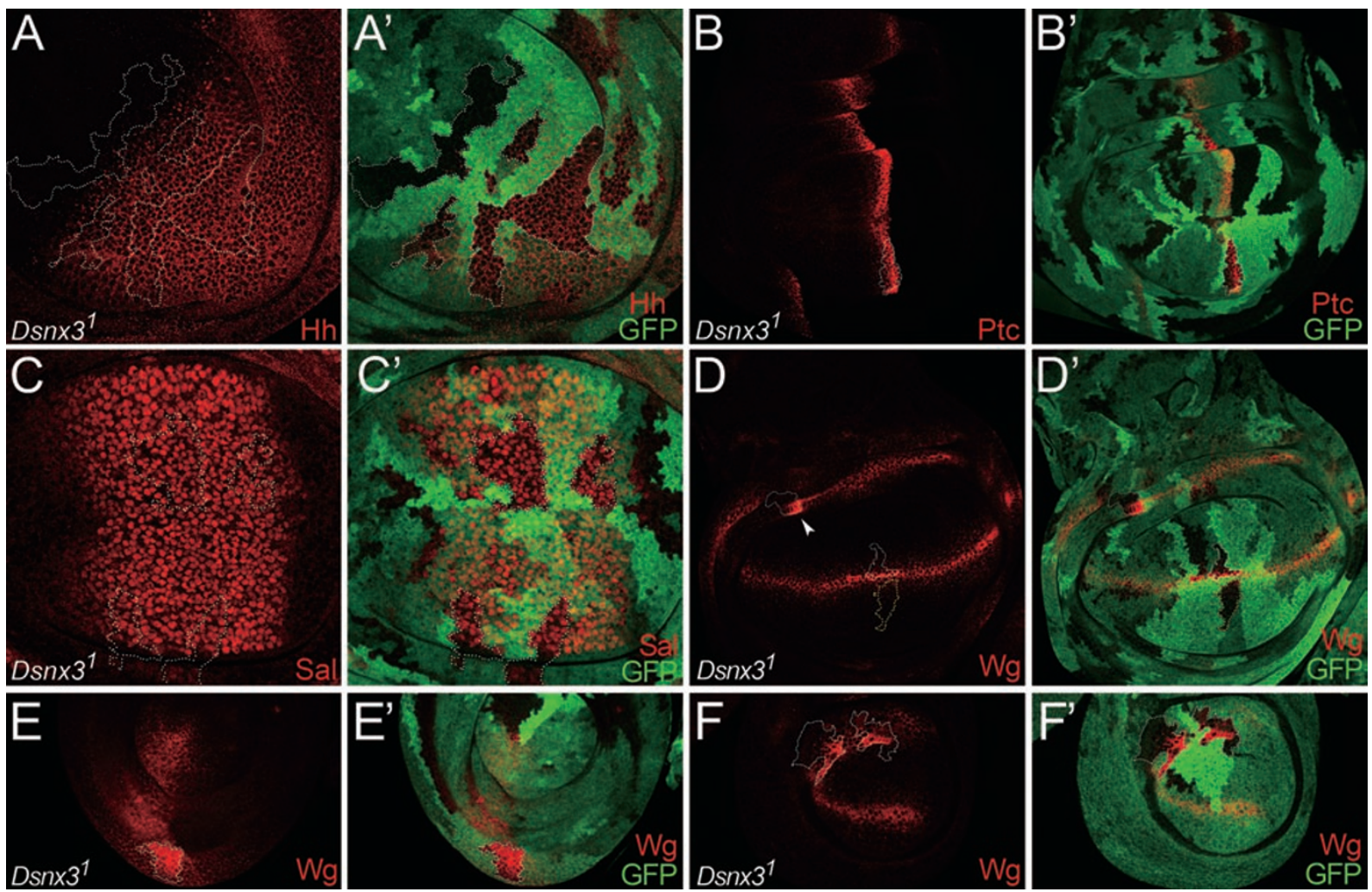

Figure 2 DSNX3 is required for Wg secretion in other tissues, but not essential for other signaling pathways. (A-A') Wing discs are oriented dorsal top-right, anterior top-left. Dsn $\times 3^{1}$ clones are marked by dotted lines and by the absence of GFP (A'). Hh protein levels (A) were altered neither in clones residing in Hh-expression cells in $\mathrm{P}$ compartment nor in those of Hhreceiving cells in A compartment. (B-D') Wing discs are oriented anterior left, dorsal up. (B-B') Wing disc bearing $D s n \times 3^{1}$ clones marked by dotted lines and by the absence of GFP (B'). Ptc (Hh receptor) levels were normal in $D s n \times 3^{1}$ mutant cells (B). (C-C') Wing disc bearing Dsn $\times 3^{1}$ mutant clones marked by dotted lines and by the absence of GFP (C'). Sal (a downstream component of Dpp signaling) levels were not altered in Dsnx3 mutant clones (C). (D-D') Wg staining in wing disc bearing $D s n \times 3^{1}$ clones marked by dotted lines and by the absence of GFP (D'). Wg protein was accumulated in its producing cells mutant for Dsnx3 but its level was not altered in a mutant clone residing outside of the Wg-producing cells marked by yellow dotted lines. Also note that $\mathrm{Wg}$ was accumulated in $D s n \times 3^{1}$ mutant $\mathrm{Wg}$-producing cells in the hinge region (arrowheads in $\mathbf{D}$ ). (E-E') Dsnx $3^{1}$ clone in leg disc (E', marked by GFP absence). As in the wing disc, Wg was accumulated in Dsnx3 mutant Wgproducing cells. (F-F') Dsn $\times 3^{1}$ clone in haltere disc (F', marked by GFP absence). As in the wing and leg discs, Wg was also accumulated in Dsnx3 mutant Wg-producing cells.

$5 \mathrm{~h}$ after induction, while significant levels of Wls protein were still maintained at this point in the wild-type cells (Supplementary information, Figure S3B-S3B"). Taken together, these data strongly argue that Wls stability is controlled by DSNX3.

We further asked whether overexpression of Wls could rescue the Wg secretion defect observed in Dsnx3 mutant cells. As shown previously [12], Wg protein levels could be enhanced in the $\mathrm{P}$ compartment expressing $U A S$-wls-HA (Figure $3 \mathrm{G}$ ). In wing discs of Dsnx3 homozygous mutant, ectopic expression of Wls using $e n^{\text {Gal4 }}$ could produce more $\mathrm{Wg}$ particles in the $\mathrm{P}$ compartment (arrowheads in Figure 3H), suggesting that overexpression of Wls can restore the $\mathrm{Wg}$ secretion defect in Dsnx3 mutant cells.

Finally, we examined whether DSNX3 and W1s formed a complex in S2 cells. V5-tagged Wls and GFPtagged DSNX3 expression vectors were transfected individually or together into S2 cells. Upon immunoprecipitation of the DSNX3-GFP protein from transfected cells, Wls-V5 was detected by western blotting in the immunoprecipitates (Figure 3I). Collectively, these data argue that DSNX3 interacts with Wls and that Wls stability is regulated by DSNX3.

\section{DSNX3 forms a complex with retromer}

Our results so far provided compelling evidence for the role of DSNX3 in regulating the stability of Wls. 

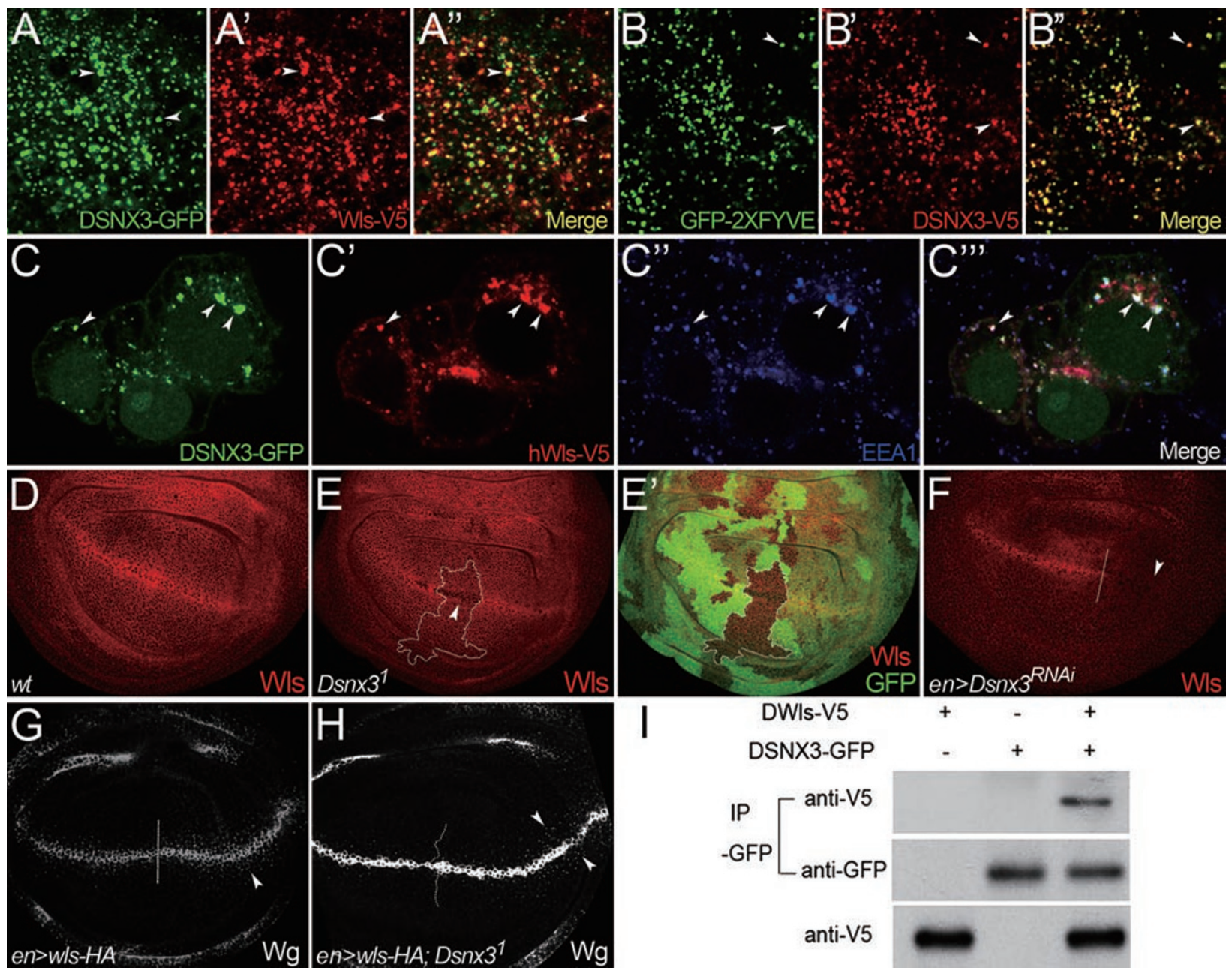

Figure 3 DSNX3 interacts with WIs and affects Wg secretion by controlling WIs stability. (A-A") UAS-Dsnx3-GFP was expressed in the $\mathrm{P}$ compartment of the wing disc using $e n^{\text {Gal }}$ in the flies carrying $h s-W / s-V 5$ construct. Wls-V5 expression was induced by heat shock for $2 \mathrm{~h}$. Another $2 \mathrm{~h}$ later, Wls-V5 protein distribution was examined. Wls-V5 levels were markedly increased in the $P$ compartment. Both V5-tagged WIs and GFP-tagged DSNX3 were detected in punctate structures (arrowheads in A and $\mathbf{A}^{\prime}$ ), and they significantly co-localized with each other (arrowheads in A"). (B-B') UAS-Dsnx3-V5 and UAS-GFP-2 $\times F Y V E$ were expressed in wing discs using en $n^{\text {Gal4 }}$. V5-tagged DSNX3 was detected in punctate structures (arrowheads in B'). DSNX3 punctate structures (B) co-localized with the early endosome marker GFP-2×FYVE (B'). Sites of co-localization are shown by arrowheads in B". (C-C'”') hWls-V5 and DSNX3-GFP expression vectors were co-transfected in HeLa cells. The subcellular localizations of hWls-V5 and DSNX3-GFP proteins were determined by immunostaining. DSNX3GFP (C, green) co-localized with hWls-V5 (C', red) in early endosomes, which were marked by the early endosome marker EEA1 (C", blue). Sites of co-localization are indicated by arrowheads in C"'. (D-F) Wing discs are oriented dorsal top-right, anterior top-left. (D) Endogenous Wls was ubiquitously expressed in the wing disc; however, Wls staining was increased at the $\mathrm{D} / \mathrm{V}$ boundary. (E-E') Endogenous Wls staining in a wing disc bearing $D s n \times 3^{1}$ clones. Mutant clones are outlined by the dotted lines to mark the absence of GFP (E', green). The accumulation of Wls in Wg-producing cells was markedly reduced in clones homozygous for $D s n \times 3^{1}$ (arrowhead in E). (F) UAS-Dsn $\times 3^{R N A i}$ was expressed in wing discs using en ${ }^{\text {Gal4 }}$ to deplete DSNX3 activity in the $\mathrm{P}$ compartment. A/P boundary is shown by the dotted line. Wls staining in the apical section of Wgproducing cells was almost absent in the P compartment (arrowhead). (G-H) Wing discs are oriented anterior left, dorsal up. A/P boundary is shown by the dotted line. (G) UAS-HA-w/s was expressed in wing discs using en ${ }^{\text {Gal4 }}$ in the $\mathrm{P}$ compartment. Note that Wg levels were somewhat enhanced when Wls was overexpressed (arrowhead). (H) UAS-w/s-HA was expressed using $e n^{\text {Gal4 }}$ in the $\mathrm{P}$ compartment of Dsnx3 homozygous mutant discs. Wg secretion appeared to be enhanced in the $\mathrm{P}$ compartment compared with the A compartment (arrowhead). (I) V5-tagged Wls and GFP-tagged DSNX3 expression vectors were transfected individually or together into S2 cells. Upon immunoprecipitation of DSNX3-GFP protein from transfected cells, Wls-V5 was detected by western blotting in the immunoprecipitates. 
Previous studies have demonstrated the importance of retromer function for Wls stability [12-14]. As SNX molecule(s) is an important component of the retromer complex, we speculated that DSNX3 either directly or indirectly controls retromer-mediated recycling of Wls. We performed co-localization experiments using HeLa cells. A GFP-tagged DSNX3 expression vector was transfected into HeLa cells, and the subcellular localization of the DSNX3-GFP protein was detected by immunostaining. As shown in Figure 4A-4A", many of the DSNX3-GFP molecules co-localized with endogenous $\mathrm{hVps} 35$ in early endosomes marked by EEA1.

We further examined whether DSNX3 formed a complex with retromer by co-immunoprecipitation assay in S2 cells. Myc-tagged DVps35 and GFP-tagged DSNX3 expression vectors were transfected individually or together into S2 cells. Upon immunoprecipitation of the DSNX3-GFP protein from transfected cells, Myc-DVps35 was detected by western blotting in the immunoprecipitates (Figure 4E). This data suggest that DSNX3 can interact with DVps35.

Finally, we further performed an epistatic analysis between DSNX3 and retromer. Enhanced levels of $\mathrm{Wg}$ were observed in the $\mathrm{P}$ or the anterior (A) compartment when DSNX3 was ectopically expressed by $e n^{\text {Gal4 }}$ or $c i^{\text {Gal4 }}$, respectively (Figure 4B-4B'", 4C and 4C'). However, when $D s n x 3$ was co-expressed with $D v p s 35^{R N A i}$ (Figure 4D and 4D') in the P compartment by en ${ }^{\text {Gal4 }}, \mathrm{Wg}$ levels were increased in the producing cells, but reduced outside of the Wg-producing cells. This data suggest that the loss of retromer activity can block the gain of function activity of DSNX3, arguing that DSNX3 acts upstream of, or in parallel with, retromer in regulating Wg secretion.

Taken together, our results demonstrate that DSNX3 interacts with DVps35 in early endosomes, supporting the view that DSNX3 is involved in retromer-mediated recycling of Wls.

\section{The PI3P-binding motif of DSNX3 in the PX domain is} essential for its function

The PX domains of the SNX3 molecules are highly conserved among SNX3 molecules from yeast to mammals (Supplementary information, Figure S4). Previous studies identified the most conserved motif in the PX domain, RR(F/Y)S(D/E)F (single letter aminoacid abbreviations are used here and hereafter), to be very sensitive to mutations $[51,52]$. Point mutations within this region of SNX3 abolished both the interaction with PI3P and membrane targeting $[51,52]$. We therefore opted to determine whether membrane targeting of
DSNX3 is essential for its activity in $\mathrm{Wg}$ secretion. We generated DSNX3(-RRY) in which all three potential PI3P attachment sites of DSNX3, R74, R75 and Y76, were converted to A74, A75 and A76, respectively. As shown earlier, overexpression of DSNX3 can promote Wg secretion (Figure 4B-B"', 4C and 4C'). Moreover, DSNX3-GFP was detected in vesicle-like punctate structures in the apical section of the $\mathrm{P}$ compartment (arrowheads in Figure 4B"'). When UAS-Dsnx3(-RRY)$G F P$ was expressed in wing discs using $e n^{\text {Galt }}$ in the $\mathrm{P}$ compartment, we observed no obvious difference in $\mathrm{Wg}$ levels between the $\mathrm{P}$ and A compartments (arrowheads in Figure 5A). The DSNX3(-RRY) was not detected in vesicle-like punctate structures, but rather accumulated throughout the cytoplasm (Figure 5A"). Furthermore, we expressed $U A S-D s n x 3(-R R Y)-G F P$ in a $D \operatorname{sn} x 3$ homozygous mutant using $e n^{\text {Gal4 }}$ in the $\mathrm{P}$ compartment. The $\mathrm{Wg}$ secretion defect cannot be rescued by the expression of Dsnx3(-RRY)-GFP (Figure 5B). Collectively, these data strongly suggest that the PI3Pbinding motif of DSNX3 is essential for the activity of DSNX3 in Wg secretion.

We further asked whether DSNX3(-RRY) lost its interaction with both W1s and Vps35. To examine this, we first performed co-localization experiments in HeLa cells. V5-tagged hWls and GFP-tagged DSNX3(RRY) expression vectors were co-transfected into HeLa cells and subcellular localizations were determined by immunostaining. DSNX3(-RRY)-GFP accumulated in the cytoplasm (Figure 5C, green), and did not colocalize with either hWls (Figure 5C', red) or the early endosome marker EEA1 (Figure 5C", blue). DSNX3(RRY)-GFP (Figure 5D, green) also did not co-localize with endogenous hVps35 (Figure 5D", blue) in early endosomes marked by EEA1(Figure 5D', red). However, when we performed co-immunoprecipitation experiments between DSNX3(-RRY) and Wls or DVps35 (Supplementary information, Figure S5), we observed the interaction between Wls and DSNX3(-RRY) (Supplementary information, Figure S5A). Similarly, DVps35 could also form a complex with DSNX3(-RRY) (Supplementary information, Figure S5B). These data indicate that the PI3P-binding site in DSNX3 is not required for the interaction of DSNX3 with either Wls or DVps35. On the basis of the above data, we suggest that the membrane targeting of DSNX3 via the PI3P binding is essential for the activity of DSNX3.

DSNX1 and DSNX6 are not essential for retromermediated $W g$ secretion

Various studies have demonstrated essential roles of SNX1/SNX2 and SNX5/SNX6 for retromer activities in 

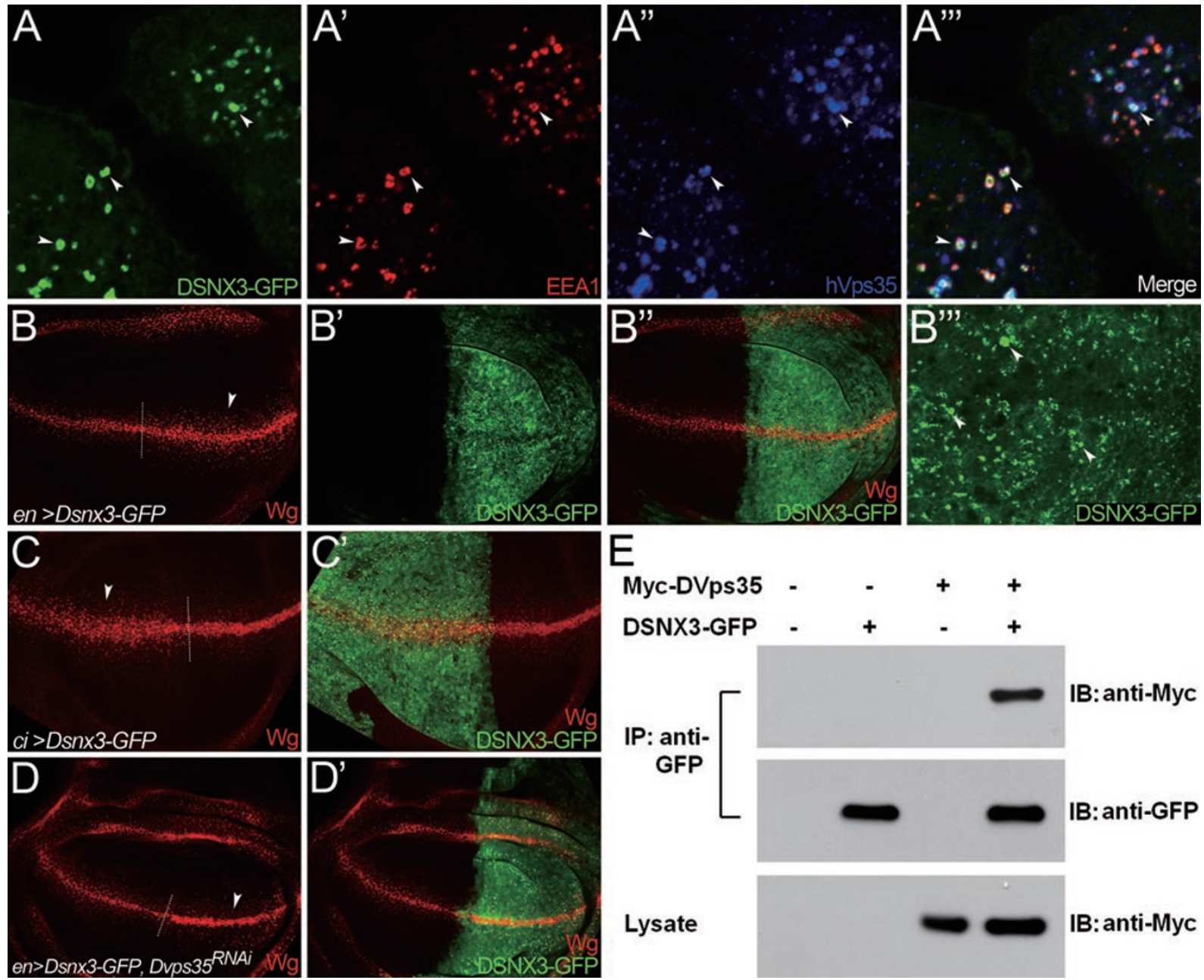

IB:anti-Myc

IB:anti-GFP

IB: anti-Myc

Figure 4 Subcellular localization and interaction of DSNX3 and Vps35. (A-A"') DSNX3-GFP expression vector was transfected into HeLa cells and DSNX3-GFP protein subcellular localization was determined by immunostaining. DSNX3GFP (A, green) co-localized with endogenous Vps35 (hVps35, blue in A') in early endosomes (arrowheads in A'") that were marked by the early endosome marker EEA1 (red in A'). (B-D') Wing discs are oriented dorsal top-right, anterior top-left. A/ $\mathrm{P}$ boundary is shown by the dotted line. (B-B'") UAS-Dsnx3-GFP was overexpressed in the $\mathrm{P}$ compartment of the wing disc using $e n^{\text {Gal4 }}$. Wg secretion was markedly expanded in the P compartment (arrowheads in B). B'"' shows the enlarged view of a segment of the posterior compartment from B'. The majority of DSNX3-GFP was detected in punctate structures in the apical section of the wing disc (arrowheads in B'"'). (C-C') UAS-Dsnx3-GFP was expressed in the A compartment of wing discs using $\mathrm{Ci}^{\mathrm{Gal}}$. Wg secretion was markedly expanded in the A compartment (arrowheads in C). (D-D') UAS-Dsnx3-GFP was co-expressed with UAS-Vps $35^{\text {RNA }}$ in the $\mathrm{P}$ compartment of wing discs using en ${ }^{\text {Gal4 }}$. Wg accumulated in Wg-producing cells of the $\mathrm{P}$ compartment and the gain of function phenotype of DSNX3 observed in B was dramatically repressed by the depletion of DVps35 (arrowhead in D). (E) Myc-tagged DVps35 and GFP-tagged DSNX3 expression vectors were co-transfected into Drosophila S2 cells. Cell lysates were immunoprecipitated and analyzed by western blotting with the antibodies indicated. IP: immunoprecipitation; IB: immunoblot.

a variety of cellular functions, including the recycling of cargo proteins Vps10p in yeast, the phagocytic receptor CED-1 in C. elegans and mammalian CI-MPR [30-33, 53]. As both $D \sin x 1$ and $D \sin x 6$ mutants are viable, they may not be essential for $\mathrm{Wg}$ secretion. In support of this view, $\mathrm{Wg}$ and Sens levels were not altered in Dsnxl mutant clones (Figure 6A-6A"). Similarly, Wg and Sens levels were also not altered when Dsnx6 is depleted by $D \operatorname{sn} x 6^{R N A i}$ (Figure 6B-6B"). Moreover, ectopic expression of DSNX1 failed to rescue $\mathrm{Wg}$ secretion defects in Dsnx3-depleted cells (Figure 6C-6C"). Similarly, ectopic expression of DSNX6 also failed to rescue $\mathrm{Wg}$ secretion defects in Dsnx3-depleted wing disc cells (Figure 6D6D") and in Dsnx3-depleted S2 cells (Figure 6E). These 

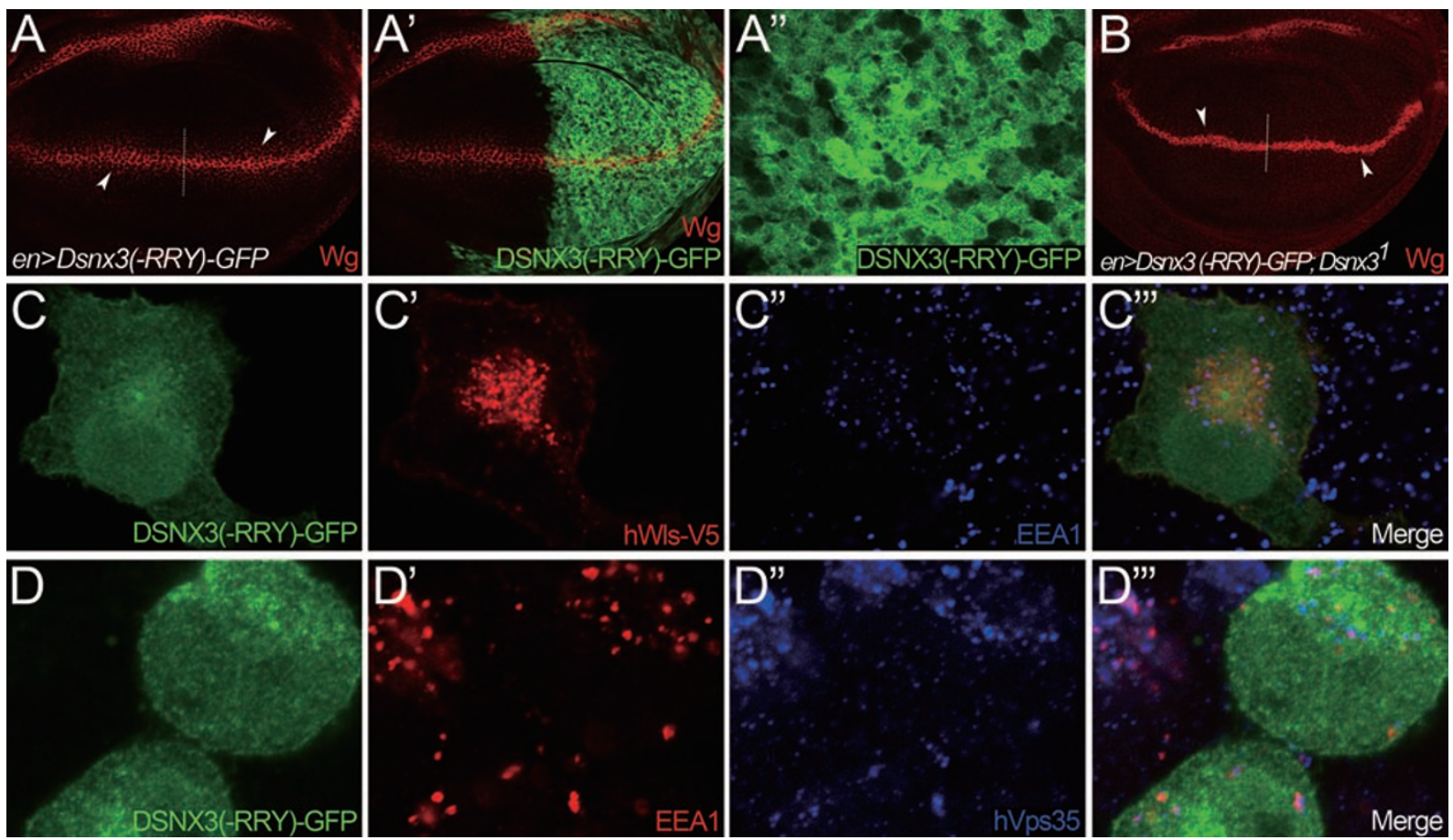

Figure 5 PI3P-binding motif of PX domain is essential for DSNX3 function. (A-B) Wing discs are oriented anterior left, dorsal up. (A-A") UAS-Dsnx3(-RRY)-GFP was expressed in the $\mathrm{P}$ compartment of wing discs using en ${ }^{\text {Gal4}}$. There was no obvious difference in $\mathrm{Wg}$ levels between the $\mathrm{P}$ and $\mathrm{A}$ compartments (arrowheads in A). DSNX3(-RRY)-GFP was distributed throughout the cytoplasm, and no vesicle-like punctate structures can be observed (A"). A" shows the enlarged view of a segment of the posterior compartment from $\mathbf{A}^{\prime}$. (B) UAS-Dsnx3(-RRY)-GFP was expressed in the $\mathrm{P}$ compartment of Dsnx3 homozygous mutant wing discs using $e n^{\text {Gal }}$. There was no detectable difference in the Wg levels between $A$ and $P$ compartments, indicating that Wg secretion defect of the Dsnx3 mutant cannot be rescued by the expression of DSNX3(RRY)-GFP. (C-C'”') hWls-V5 and DSNX3(-RRY)-GFP expression vectors were co-transfected into HeLa cells. The subcellular localization of hWls-V5 and DSNX3(-RRY)-GFP proteins were determined by immunostaining. DSNX3(-RRY)-GFP was distributed in the cytoplasm (C, green), and lost the co-localization with hWls-V5 (C', red) and the early endosome marker EEA1 (C"', blue). (D-D'”) DSNX3(-RRY)-GFP expression vector was transfected into HeLa cells. DSNX3(-RRY)-GFP protein was detected in the cytoplasm (D, green) and did not co-localized with early endosome marker EEA1 (D', red) and hVps35 (D', blue).

data argue that the activity of DSNX3 in retromermediated Wg secretion cannot be replaced by DSNX1 and/or DSNX6, further suggesting the specificity of DSNX3 in Wg secretion.

\section{Discussion}

\section{Roles of DSNX3 in Wg secretion}

A main finding in this work is the demonstration that DSNX3 controls Wg secretion by regulating retromermediated Wls recycling. First, we showed that the loss of DSNX3 activity in the wing disc caused increased levels of $\mathrm{Wg}$ inside the producing cells, but reduced levels in the receiving cells. Cell culture experiments further demonstrated that secreted $\mathrm{Wg}$ in the conditioned medium was reduced when $\mathrm{Wg}$-producing S2 cells were treated with $D \sin x 3$ dsRNA. These data provide compelling evidence for a critical role of DSNX3 in $\mathrm{Wg}$ secretion. Second, we showed that the Wls level was strikingly reduced in the wing disc in the absence of DSNX3 activity. We further showed that DSNX3 colocalized with Wls and can be co-immunoprecipitated with Wls. Similarly, DSNX3 also co-localized with Vps35 and can be co-immunoprecipitated with Vps35. Thus, this work extends further our previous findings of a critical role for retromer in $\mathrm{Wg} / \mathrm{Wnt}$ secretion by linking SNX3 into this regulatory pathway.

Interestingly, although DSNX3 is essential for $\mathrm{Wg}$ secretion, we observed no obvious defects in Dpp or $\mathrm{Hh}$ signaling. Previous data showed that loss of retromer activity did not affect Dpp or Hh signaling. In this regard, DSNX3 recapitulates the defects associated with retromer 

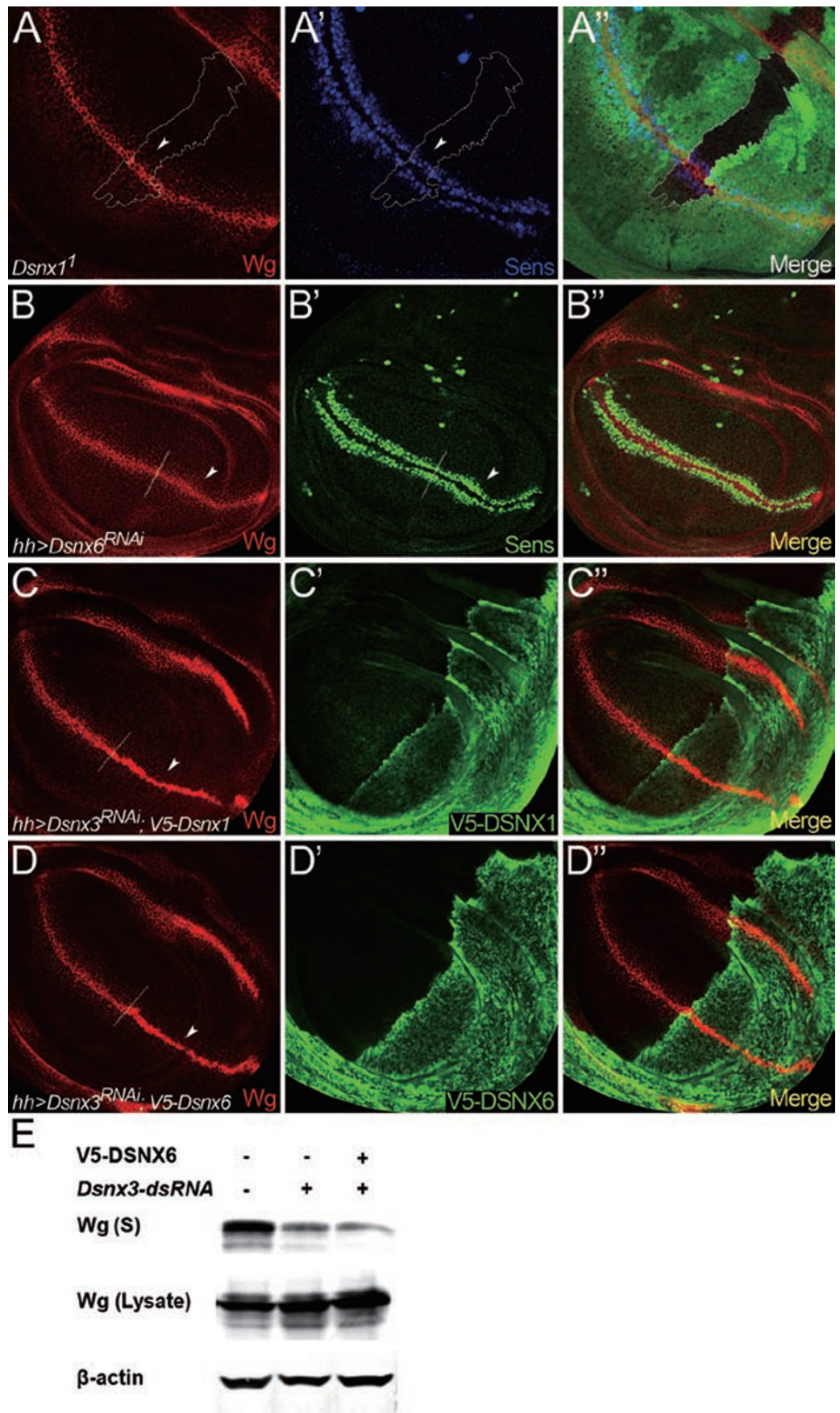

Figure 6 DSNX1 and DSNX6 are not required for Wg secretion and signaling. (A-C) Wing discs are oriented dorsal topright, anterior top-left. (A-A") Wg and Sens staining in a wing disc bearing Dsnx $1^{1}$ clones. Mutant clone is outlined by the dotted lines to mark the absence of GFP (A", green). Wg levels were not altered in the absence of Dsnx1 (arrowhead in A). Sens staining was also not altered in the mutant clone (arrowhead in $\mathbf{A}^{\prime}$ ). (B-B') UAS-Dsnx6 ${ }^{R N A i}$ was expressed in wing discs using $h h^{\text {Gal4 }}$ to deplete Dsnx6 activity in the $\mathrm{P}$ compartment. A/P boundary is shown by the dotted line. Wg was secreted normally under these conditions (arrowhead in B), and the expression of Sens was also not reduced (arrowhead in B'). (CC") UAS-Dsn $\times 3^{R N A i}$ and UAS-V5-Dsnx1 were co-expressed in the $\mathrm{P}$ compartment using $h h^{\text {Gal }}$. Wg accumulated in the $\mathrm{P}$ compartment (arrowhead in C), indicating that the Wg secretion defect caused by DSNX3 depletion cannot be rescued by the overexpression of DSNX1. (D-D”) UAS-Dsnx $3^{\text {RNAi }}$ and UAS-V5-Dsnx6 were co-expressed in the P compartment using $h h^{\text {Gal4 }}$. Wg accumulated in the $\mathrm{P}$ compartment (arrowhead in D), indicating that the Wg secretion defect caused by DSNX3 depletion cannot be rescued by the overexpression of DSNX6. (E) The amount of Wg in the supernatant (S) was strongly reduced when $\mathrm{S} 2+\mathrm{pMK}-\mathrm{Wg}$ cells were treated with dsRNAs targeted to the coding region of Dsnx3. However, the Wg secretion defect caused by DSNX3 depletion cannot be rescued by the overexpression of DSNX6. 
activity. On the other hand, the Dvps35 mutant is lethal, but the Dsnx 3 mutant is viable. In addition, Dvps 35 homozygous mutant larvae exhibited other defects such as melanotic tumor formation $[13,54]$. However, homozygous Dsnx3 mutant larvae did not exhibit any detectable melanotic tumors (data not shown). These data suggests that SNX molecule(s) other than DSNX3 are involved in other retromer-mediated activities. On the basis of the above observations, we propose that DSNX3 forms a specific retromer subcomplex that is required for $\mathrm{Wls}$ recycling and $\mathrm{Wg}$ secretion.

While DSNX3 is essential for Wg secretion, one important issue that remains to be resolved is whether DSNX3 is required for all retromer-mediated Wls recycling and $\mathrm{Wg}$ secretion. In addition to wing discs, we also observed $\mathrm{Wg}$ secretion defects in other tissues, including leg and haltere discs. Similarly, in cultured S2 cells, DSNX3 is critical for Wg secretion, as depletion of $D \operatorname{sn} x 3$ by RNAi in cultured $\mathrm{S} 2$ cells also caused a $\mathrm{Wg}$ secretion defect. However, both wls and Dvps35 null mutant embryos are lethal, while Dsnx3 null embryos are viable. It is important to mention that Dsnx3 mutant had relatively weaker $\mathrm{Wg}$ secretion defects than $w l s$ and Dvps 35 mutants. We also found that Dsnx3 mutant embryos derived from $D s n \times 3$ homozygous females were normal, arguing that $\mathrm{Wg}$ secretion in the $D \operatorname{sn} x 3$ mutant embryos is not defective (data not shown). These data suggest a possibility that mechanisms other than DSNX3-mediated Wls recycling might be involved in retromer-mediated $\mathrm{Wg}$ secretion in the embryos, and possibly in other tissues. Further experiments are needed to determine such mechanisms.

Specific role of DSNX3 in the function of the retromer complex in $\mathrm{Wg}$ secretion

Another important observation of this work is the specificity of DSNX3 in retromer-mediated Wls recycling. Various studies have demonstrated the importance of SNX1/SNX2 and SNX5/SNX6 in diverse functions of retromer, including retrograde transport of yeast Vps10p, the C. elegans phagocytic receptor CED-1 and the mammalian CI-MPR [30-33, 53]. Therefore, DSNX3 is an atypical SNX molecule involved in the activity of retromer in $\mathrm{Wg}$ secretion. We showed that $\mathrm{Wg}$ secretion was normal in the absence of either DSNX1 or DSNX6 activity, arguing that DSNX1 and DSNX6 are not essential for Wg secretion. Importantly, we found that the activity of DSNX3 in Wg secretion cannot be simply replaced by overexpression of DSNX1 or DSNX6, further demonstrating that the specific activity of SNX3, rather than SNX1/SNX6, is involved in retromer-mediated $\mathrm{Wg}$ secretion. Among SNX molecules, a unique feature of SNX3 is the lack of a BAR domain, which has been shown to be essential for dimerization and membrane binding. The BAR domain preferentially interacts with membranes of positive curvature, drives membrane deformation and generates membrane tubules [55]. Thus, one possibility is that a specific structural property of DSNX3 allows it to form a subcomplex with retromer that is distinct from other classic SNX-BAR-retromer complexes. Alternatively, as DSNX3 can interact with Wls, the association of DSNX3 with Wls may allow Wls to load onto the SNX3-retromer complex for retrograde transport. In this case, the function of DSNX3 is very similar to that of Grd19p, the yeast SNX3 that interacts with the cargo iron transporter Fet3p-Ftr1p, and regulates retromer-dependent retrieval of Fet3p-Ftr1p [36-38]. Our results pinpoint that the specificity of retromer function can be attributed to SNX molecules.

During preparation of our manuscript, a paper from Cullen, Basler and Korswagen groups was published, in which they reported the identification of SNX3 as an essential retromer component involved in Wnt secretion [56]. Both their and our independent observations reach the same conclusion that SNX3, rather than SNX1 and SNX6, is required for $\mathrm{Wg} / \mathrm{Wnt}$ secretion and $\mathrm{Wls}$ recycling. In their study, they showed that $C$. elegans devoid of both SNX1 and SNX6 activities exhibited no defect in Wnt secretion [56]. Consistent with their data, we also observed no Wg secretion defects in Drosophila Dsnx 1 mutants and in Dsnx6 RNAi-depleted cells. Importantly, we showed that overexpression of either DSNX1 or DSNX6 failed to rescue Wg secretion defects observed in Dsnx 3 mutant cells. These data suggest qualitative differences between SNX3-mediated retromer activity in $\mathrm{Wg} / \mathrm{Wnt}$ secretion and SNX1/SNX6-mediated retromer activity in other cellular processes. Moreover, we observed specific interactions of DSNX3 with Wls in co-immunoprecipitation experiments, and such interactions are independent of the PI3P-binding motif in the PX domain of DSNX3, while their experiments failed to detect a Wls-SNX3 interaction. Further studies are needed to define detailed mechanisms by which SNX3 regulates Wls recycling in development.

\section{Materials and Methods}

\section{Drosophila stocks}

The following fly lines were used in this study: the transgenic lines: $U A S-w l s-H A, U A S-G F P-2 \times F Y V E$ and $h s-w l s-V 5$ were described previously [12]. en ${ }^{\text {Gal4 }}, h h^{\text {Gal4 }}$ and $W g^{\text {LacZ }}$ were as described in FlyBase. vg Q1206-Gal4 UAS-Flp [41] was used to generate mosaic clones mutant for $D s n \times 3^{l}$ in developing wings. The following RNAi lines were obtained from the Vienna Drosophila RNAi Center: $U A S-D s n x 3^{R N A i}(104494)$ and $U A S-D \operatorname{sn} x 6^{R N A i}$ (24276). 
The Dsnx1 (CG2774) mutant was generated by imprecise excision of a P element (Bloomington stock number 27118). The mutant has a 1 566-bp deletion starting from $130 \mathrm{bp}$ upstream of the Dsnxl ATG start codon and ending in the middle of the third exon, and therefore is likely to be a null allele. UAS-Dsnx3-V5, UAS-Dsnx3GFP, UAS-Dsnx3(-RRY)-GFP, UAS-V5-Dsnx1 and UAS-V5-Dsnx6 transgenic lines were generated in this study.

\section{Generation of the Dsnx3 null allele, Dsnx $3^{1}$}

The Dsnx3 (CG6359) gene is located on the right arm of the third chromosome. We identified a $\mathrm{P}$ element insertion $D \operatorname{sn} x 3^{E Y}$ (EY05688) [57] that resides in the first exon of the Dsnx3 gene and is a semi-lethal allele (data not shown). After mobilization of the EY05688 P element, we screened for Dsnx3 deletions and obtained two Dsn $x 3^{l}$ null alleles, Dsn $x 3^{l}$ and Dsn $x 3^{2} . D \operatorname{sn} x 3^{l}$ had 1398 bp deletion that included the start codon and all the three exons. $D \operatorname{sn} x 3^{2}$ deletion was 1659 bp and was extended even further in $3^{\prime} \mathrm{UTR}$ of the $D \operatorname{sn} x 3$ gene. $D \operatorname{sn} x 3^{1}$ was used for all of the experiments in this study.

\section{Generation of mutant clones and ectopic expression experiments}

Clones of mutant cells were generated by the FLP-FRT method [46] and induced in first/second-instar larvae by heat shock at $37{ }^{\circ} \mathrm{C}$ for $2 \mathrm{~h}$. We list the genotypes and conditions used in our analyses below:

(1) $D \operatorname{sn} x 3^{1}$ clones marked by absence of GFP:

$y$ w hsp70-flp/+ or Y; FRT ${ }^{82 B}$ ubiquitin-GFP/FRT $T^{82 B}$ Dsn $x 3^{l}$ (Figures 1B-1B'", 1C and 1C', 2A-2F', 3E and 3E'; Supplementary information, Figure S2A and S2A').

$y$ w hsp70-flp/+ or $Y ; \mathrm{Wg}^{\mathrm{LacZ} /+} ; \mathrm{FRT}^{82 B}$ ubiquitin-GFP/FRT $T^{82 B}$ $\operatorname{Dsnx} 3^{l}$ (Figure 1G-1G').

$y$ whp h0-flp/+ or $Y$; hs-wls-V5/+; FRT $T^{82 B}$ ubiquitin-GFP/ $F R T^{82 B} D \operatorname{sn} x 3^{1}$ (Supplementary information, Figure S3B and S3B").

(2) Wing disc expression of $U A S-D \operatorname{sn} x 3^{R N A i}$ using $e n^{\text {Gal4 }}$ or $h h^{\text {Gal4 }}$ :

$e n^{G a l 4} / U A S-D s n \times 3^{R N A i}$ (Figures $1 \mathrm{D}, 1 \mathrm{E}$ and $3 \mathrm{~F}$ ), UAS$D s n \times 3^{R N A i} /+; h h^{\text {Gal4 }}$ (Supplementary information, Figure S3AS3A"), UAS-Dsnx $3^{\text {RNAi }} /+$; $h h^{\text {Gal4 } / V 5-D s n x 1 ~(F i g u r e ~ 6 C-6 C "), ~ U A S-~}$ $D s n x 3^{R N A i} /+; h h^{\text {Gal4 }} / V 5-D \operatorname{sn} x 6$ (Figure 6D-6D”).

(3) $D \operatorname{sn} x 3^{l}$ rescue experiments:

en ${ }^{\text {Gal4 }} U A S-G F P / U A S-D \operatorname{sn} x 3-V 5 ; D \operatorname{sn} \times 3^{1} / D \operatorname{sn} x 3^{l}$ (Figure 1F)

$e n^{\text {Gal4 }} / U A S-w l s-H A ; D s n \times 3^{l} / D s n x 3^{l}$ (Figure $3 \mathrm{H}$ )

$e n^{\text {Gal4 }} / D \operatorname{sn} x 3(-R R Y)-G F P$; $D \operatorname{sn} x 3^{l} / D \operatorname{sn} x 3^{l}$ (Figure 5B)

(4) Co-localization of Wls with DSNX3:

en ${ }^{\text {Gal4 }} / U A S-D s n \times 3-G F P ; h s-W l s-V 5 /+$ (Figure 3A-3A")

(5) Co-localization of DSNX3 with early endosome marker:

$e^{\text {Gal4 }} / U A S-D s n x 3-V 5$; UAS- GFP-2 $\times F Y V E /+$ (Figure 3B-3B")

(6) $D \operatorname{snx} l^{l}$ clones marked by absence of GFP (Figure 6A-6A")

$y$ w hsp 70-flp/+ or Y; FRT $T^{40 A}$ ubiquitin-GFP/FRT $T^{40 A} D s n x 1^{1}$

(7) Epistatic analysis of DSNX3 and Vps35 (Figure 4D-4D') en ${ }^{\text {Gal4 }} / U A S-v p s 35^{R N A i} ; U A S-D s n x 3-G F P /+$

(8) Generation of $D \operatorname{sn} x 3^{l}$ mosaic clones mutant in developing wings using "direct mosaic system" (Supplementary information, Figure S1B and S1B")

vg Q1206-Gal4 UAS-Flp/+; FRT $T^{82 B} / F R T^{82 B} D \operatorname{sn} x 3^{l}$

(9) Wing disc expression of $U A S-D \operatorname{sn} x 6^{R N A i}$ using $h h^{\text {Gall }}$ :

$h h^{\text {Gal4 }} / U A S-D \operatorname{sn} x 6^{R N A i}$ (Figure 6B-6B")
Antibodies used for immunostaining, immunoprecipitation and western blotting

Antibody staining of wing imaginal discs or cells was performed using standard protocols. The following primary antibodies were used: mouse anti-Wg (4D4; DSHB), guinea pig anti-Sens [44], rabbit anti-Wls (made in our laboratory according to [14]), rabbit anti-GFP Alexa Fluor 488 (Molecular Probe), mouse anti-lacZ (Abmart), mouse anti-V5 (Invitrogen), rabbit anti-V5 (Sigma), mouse anti-EEA1 (BD Biosciences), goat antihVps35 (IMGENEX), rabbit anti-Hh [58], mouse anti-Ptc (DSHB) and rabbit anti-Sal (made in our laboratory). The primary antibodies were detected by fluorescence-conjugated secondary antibodies from Jackson ImmunoResearch Laboratories, Inc. Confocal fluorescence imaging was performed with a Zeiss LSM 780 laserscanning microscope (Carl Zeiss).

The primary antibodies used for immunoprecipitation and western blot analysis were rabbit anti-V5 (Sigma), guinea pig antiGFP (made in our laboratory), mouse anti-V5 (Invitrogen), mouse anti-Wg (4D4; DSHB), mouse anti-Myc (Invitrogen) and mouse anti- $\beta$-actin (Abmart).

\section{Transgenes and dsRNA generation}

To generate V5 or GFP C-terminally tagged DSNX3, the complete Dsnx3 coding sequence fragment was amplified from $D s n x 3$ cDNA clone RE52028 and inserted into $p U A S T-V 5-$ $6 x H i s$ and $p U A S T-E G F P$ vectors (established by our laboratory), respectively. $D \operatorname{sn} x 3(-R R Y)$ was generated using Invitrogen's Genetailor mutagenesis kit. All three potential PI3P-binding sites, R74, R75 and Y76 were converted to A74, A75 and A76, respectively. After that the mutated Dsnx 3 fragment was inserted into $p U A S T$ EGFP vector.

The dsRNAs for Drosophila S2 cells were generated from Dsnx3 cDNA using MEGAscript in vitro transcription kit from Ambion according to the protocol as described (http://flyrnai.org/ all_protocols.html).

The RNAil dsRNA was generated using the primers:

5'-TAATACGACTCACTATAGGG-ggetgcgaaacgagetggag-3'

5'-TAATACGACTCACTATAGGG-aatccgggcaagcacacacatt-3'

The RNAi2 dsRNA was generated using the primers:

5'-TAATACGACTCACTATAGGG-tttagaccaatctgccegtc-3' 5'-TAATACGACTCACTATAGGG-tttcctcttaaggattgaaacttt-3'

The control (GFP) dsRNA was generated from GFP cDNA using the primers:

5'-TAATACGACTCACTATAGGG G-acgtaaacggccacaagtt-3' 5'-TAATACGACTCACTATAGGG-tgttctgctggtagtggtcg-3'

Cell culture, transfection, co-immunoprecipitation and western blotting

Drosophila S2 cells were maintained at $25^{\circ} \mathrm{C}$ in HyQ SFXINSECT cell culture medium (Hyclone SH30278.01). Drosophila $\mathrm{S} 2 \mathrm{R}+$ cells were maintained at $25{ }^{\circ} \mathrm{C}$ in Schneider's medium (Gibco) supplemented with 10\% fetal bovine serum (FBS, Gibco). HeLa cells were maintained in Dulbecco's Modified Eagle Medium (Gibco) supplemented with 10\% FBS.

For co-immunoprecipitation experiments, S2 cells were transfected using Effectene transfection reagent (QIAGEN) in $100 \mathrm{~mm}$ dishes with $4 \mu \mathrm{g}$ of total DNA, including $p U A S T-D \operatorname{sn} x 3-$ GFP, pUAST-Dwls-V5 (or pUAST-Myc-Dvps35) and pArmadilloGal4. For HeLa cells' transfection, we used Polyfect transfection 
reagent (QIAGEN) in the same experimental conditions. Cells were harvested $60 \mathrm{~h}$ later and lysed in $900 \mathrm{ml}$ of $150 \mathrm{mM} \mathrm{NaCl}$, $20 \mathrm{mM}$ Tris- $\mathrm{HCl} \mathrm{pH}$ 7.5, $1.5 \%$ Triton X-100, 1 mM EDTA plus proteinase inhibitors (Roche) on ice for $1 \mathrm{~h}$. After pre-clearance with protein G Sepharose 4 Fast Flow (Amersham) beads, the lysates were incubated with guinea pig anti-GFP (made in our laboratory) for $4 \mathrm{~h}$ at $4{ }^{\circ} \mathrm{C}$, and then incubated for additional $2 \mathrm{~h}$ in the presence of $20 \mu \mathrm{l}$ of beads. Beads were washed four times with lysis buffer, and eluted in Laemmli sample buffer. Eluted samples were analyzed by western blotting. The primary antibodies used for western blot are rabbit anti-GFP (Abmart), mouse anti-V5 (Invitrogen) and mouse anti-Myc (Invitrogen).

\section{RNAi and Wg secretion assays}

To examine the effect of $D \operatorname{sn} x 3$ RNAi on Wg secretion, $5 \times 10^{6}$ $\mathrm{S} 2 \mathrm{R}+$ cells were seeded in a $60 \mathrm{~mm}$ dish and transfected the next day with $2 \mu \mathrm{g}$ of $p M K 33-W g$, using Effectene (QIAGEN). At 24 $\mathrm{h}$ post transfection, cells were sub-seeded into six-well plates at a concentration of $2 \times 10^{6}$ cells/well in $0.5 \mathrm{ml}$ serum-free media with $45 \mu \mathrm{g}$ of Dsnx 3 dsRNA and incubated at $25^{\circ} \mathrm{C}$ for $1 \mathrm{~h}$, then $0.5 \mathrm{ml}$ of serum-containing medium was added. The remaining procedure was as described $[10,12]$.

\section{Acknowledgments}

We thank Dr Hugo J Bellen (Baylor College of Medicine, USA), Dr Susan Cumberledge (University of Massachusetts, USA) and the Iowa Developmental Studies Hybridoma Bank (IDSHB) for antibodies; the Bloomington Stock Center and the Vienna Drosophila RNAi Center for Drosophila stocks. This work was supported by grants from the National Basic Research Program of China (2011CB943901), the National Natural Science Foundation of China (31030049 and 31071284), the Chinese Academy of Sciences (KSCX2-YW-R-263), and by NIH grants (2R01 GM063891 and 1R01GM087517).

\section{References}

1 MacDonald BT, Tamai K, He X. Wnt/beta-catenin signaling: components, mechanisms, and diseases. Dev Cell 2009; 17:926.

2 Clevers H. Wnt/beta-catenin signaling in development and disease. Cell 2006; 127:469-480.

3 Klaus A, Birchmeier W. Wnt signalling and its impact on development and cancer. Nat Rev Cancer 2008; 8:387-398.

4 Moon RT, Bowerman B, Boutros M, Perrimon N. The promise and perils of Wnt signaling through beta-catenin. Science 2002; 296:1644-1646.

5 Wodarz A, Nusse R. Mechanisms of Wnt signaling in development. Annu Rev Cell Dev Biol 1998; 14:59-88.

6 Tabata T, Takei Y. Morphogens, their identification and regulation. Development 2004; 131:703-712.

7 Vincent JP, Dubois L. Morphogen transport along epithelia, an integrated trafficking problem. Dev Cell 2002; 3:615-623.

8 Port F, Basler K. Wnt trafficking: new insights into Wnt maturation, secretion and spreading. Traffic 2010; 11:1265-1271.

9 Banziger C, Soldini D, Schutt C, Zipperlen P, Hausmann G, Basler K. Wntless, a conserved membrane protein dedicated to the secretion of Wnt proteins from signaling cells. Cell 2006; 125:509-522.

10 Bartscherer K, Pelte N, Ingelfinger D, Boutros M. Secretion of Wnt ligands requires Evi, a conserved transmembrane protein. Cell 2006; 125:523-533.

11 Goodman RM, Thombre S, Firtina Z, et al. Sprinter: a novel transmembrane protein required for $\mathrm{Wg}$ secretion and signaling. Development 2006; 133:4901-4911.

12 Belenkaya TY, Wu Y, Tang X, et al. The retromer complex influences Wnt secretion by recycling wntless from endosomes to the trans-Golgi network. Dev Cell 2008; 14:120-131.

13 Franch-Marro X, Wendler F, Guidato S, et al. Wingless secretion requires endosome-to-Golgi retrieval of Wntless/Evi/ Sprinter by the retromer complex. Nat Cell Biol 2008; 10:170177.

14 Port F, Kuster M, Herr P, et al. Wingless secretion promotes and requires retromer-dependent cycling of Wntless. Nat Cell Biol 2008; 10:178-185.

15 Fu J, Ivy Yu HM, Maruyama T, Mirando AJ, Hsu W. Gpr177/ mouse Wntless is essential for Wnt-mediated craniofacial and brain development. Dev Dyn 2011; 240:365-371.

16 Fu J, Jiang M, Mirando AJ, Yu HM, Hsu W. Reciprocal regulation of Wnt and Gpr177/mouse Wntless is required for embryonic axis formation. Proc Natl Acad Sci USA 2009; 106:18598-18603.

17 Pan CL, Baum PD, Gu M, Jorgensen EM, Clark SG, Garriga G. C. elegans AP-2 and retromer control Wnt signaling by regulating mig-14/Wntless. Dev Cell 2008; 14:132-139.

18 Yang PT, Lorenowicz MJ, Silhankova M, Coudreuse DY, Betist MC, Korswagen HC. Wnt signaling requires retromerdependent recycling of MIG-14/Wntless in Wnt-producing cells. Dev Cell 2008; 14:140-147.

19 Coudreuse DY, Roel G, Betist MC, Destree O, Korswagen $\mathrm{HC}$. Wnt gradient formation requires retromer function in Wnt-producing cells. Science 2006; 312:921-924.

20 Prasad BC, Clark SG. Wnt signaling establishes anteroposterior neuronal polarity and requires retromer in C. elegans. Development 2006; 133:1757-1766.

21 Bonifacino JS, Hurley JH. Retromer. Curr Opin Cell Biol 2008; 20:427-436.

22 Seaman MN. Recycle your receptors with retromer. Trends Cell Biol 2005; 15:68-75.

23 McGough IJ, Cullen PJ. Recent advances in retromer biology. Traffic 2011; 12:963-971.

24 Cullen PJ. Endosomal sorting and signalling: an emerging role for sorting nexins. Nat Rev Mol Cell Biol 2008; 9:574-582.

25 Seet LF, Hong W. The Phox (PX) domain proteins and membrane traffic. Biochim Biophys Acta 2006; 1761:878-896.

26 Teasdale RD, Loci D, Houghton F, Karlsson L, Gleeson PA. A large family of endosome-localized proteins related to sorting nexin 1. Biochem J 2001; 358:7-16.

27 Rojas R, Kametaka S, Haft CR, Bonifacino JS. Interchangeable but essential functions of SNX1 and SNX2 in the association of retromer with endosomes and the trafficking of mannose 6-phosphate receptors. Mol Cell Biol 2007; 27:11121124.

28 Griffin CT, Trejo J, Magnuson T. Genetic evidence for a mammalian retromer complex containing sorting nexins 1 and 2 . Proc Natl Acad Sci USA 2005; 102:15173-15177. 
29 Wassmer T, Attar N, Bujny MV, Oakley J, Traer CJ, Cullen PJ. A loss-of-function screen reveals SNX5 and SNX6 as potential components of the mammalian retromer. J Cell Sci 2007; 120:45-54.

30 Chen D, Xiao H, Zhang K, et al. Retromer is required for apoptotic cell clearance by phagocytic receptor recycling. Science 2010; 327:1261-1264.

31 Seaman MN. Identification of a novel conserved sorting motif required for retromer-mediated endosome-to-TGN retrieval. $J$ Cell Sci 2007; 120:2378-2389.

32 Seaman MN. Cargo-selective endosomal sorting for retrieval to the Golgi requires retromer. J Cell Biol 2004; 165:111-122.

33 Arighi CN, Hartnell LM, Aguilar RC, Haft CR, Bonifacino JS. Role of the mammalian retromer in sorting of the cationindependent mannose 6-phosphate receptor. J Cell Biol 2004; 165:123-133.

34 Hong Z, Yang Y, Zhang C, et al. The retromer component SNX6 interacts with dynactin p150(Glued) and mediates endosome-to-TGN transport. Cell Res 2009; 19:1334-1349.

35 Wassmer T, Attar N, Harterink M, et al. The retromer coat complex coordinates endosomal sorting and dynein-mediated transport, with carrier recognition by the trans-Golgi network. Dev Cell 2009; 17:110-122.

36 Strochlic TI, Setty TG, Sitaram A, Burd CG. Grd19/Snx3p functions as a cargo-specific adapter for retromer-dependent endocytic recycling. J Cell Biol 2007; 177:115-125.

37 Zhou CZ, Li de La Sierra-Gallay I, Quevillon-Cheruel S, et al. Crystal structure of the yeast Phox homology (PX) domain protein Grd19p complexed to phosphatidylinositol-3-phosphate. J Biol Chem 2003; 278:50371-50376.

38 Voos W, Stevens TH. Retrieval of resident late-Golgi membrane proteins from the prevacuolar compartment of Saccharomyces cerevisiae is dependent on the function of Grd19p. $J$ Cell Biol 1998; 140:577-590.

39 Temkin P, Lauffer B, Jager S, Cimermancic P, Krogan NJ, von Zastrow M. SNX27 mediates retromer tubule entry and endosome-to-plasma membrane trafficking of signalling receptors. Nat Cell Biol 2011; 13:717-723.

40 Gong WJ, Golic KG. Ends-out, or replacement, gene targeting in Drosophila. Proc Natl Acad Sci USA 2003; 100:2556-2561.

41 Belenkaya TY, Han C, Standley HJ, Lin X, Houston DW, Heasman J. pygopus Encodes a nuclear protein essential for wingless/Wnt signaling. Development 2002; 129:4089-4101.

42 Couso JP, Bishop SA, Martinez Arias A. The wingless signalling pathway and the patterning of the wing margin in Drosophila. Development 1994; 120:621-636.

43 Neumann CJ, Cohen SM. Long-range action of Wingless organizes the dorsal-ventral axis of the Drosophila wing. Development 1997; 124:871-880.

44 Nolo R, Abbott LA, Bellen HJ. Senseless, a Zn finger transcription factor, is necessary and sufficient for sensory organ development in Drosophila. Cell 2000; 102:349-362.
45 Zecca M, Basler K, Struhl G. Direct and long-range action of a wingless morphogen gradient. Cell 1996; 87:833-844.

$46 \mathrm{Xu} \mathrm{T}$, Rubin GM. Analysis of genetic mosaics in developing and adult Drosophila tissues. Development 1993; 117:12231237.

47 Strigini M, Cohen SM. Wingless gradient formation in the Drosophila wing. Curr Biol 2000; 10:293-300.

48 Rulifson EJ, Blair SS. Notch regulates wingless expression and is not required for reception of the paracrine wingless signal during wing margin neurogenesis in Drosophila. Development 1995; 121:2813-2824.

49 Gaullier JM, Simonsen A, D'Arrigo A, Bremnes B, Stenmark H, Aasland R. FYVE fingers bind PtdIns(3)P. Nature 1998; 394:432-433.

50 Christoforidis S, McBride HM, Burgoyne RD, Zerial M. The Rab5 effector EEA1 is a core component of endosome docking. Nature 1999; 397:621-625.

$51 \mathrm{Xu}$ Y, Seet LF, Hanson B, Hong W. The Phox homology (PX) domain, a new player in phosphoinositide signalling. Biochem $J$ 2001; 360:513-530.

$52 \mathrm{Xu}$ Y, Hortsman H, Seet L, Wong SH, Hong W. SNX3 regulates endosomal function through its PX-domain-mediated interaction with PtdIns(3)P. Nat Cell Biol 2001; 3:658-666.

53 Seaman MN, McCaffery JM, Emr SD. A membrane coat complex essential for endosome-to-Golgi retrograde transport in yeast. J Cell Biol 1998; 142:665-681.

54 Korolchuk VI, Schutz MM, Gomez-Llorente C, et al. Drosophila Vps35 function is necessary for normal endocytic trafficking and actin cytoskeleton organisation. J Cell Sci 2007; 120:4367-4376.

55 Gallop JL, McMahon HT. BAR domains and membrane curvature: bringing your curves to the BAR. Biochem Soc Symp 2005; 72:223-231.

56 Harterink M, Port F, Lorenowicz MJ, et al. A SNX3-dependent retromer pathway mediates retrograde transport of the Wnt sorting receptor Wntless and is required for Wnt secretion. Nat Cell Biol 2011; 13:914-923.

57 Bellen HJ, Levis RW, Liao G, et al. The BDGP gene disruption project: single transposon insertions associated with $40 \%$ of Drosophila genes. Genetics 2004; 167:761-781.

58 Taylor AM, Nakano Y, Mohler J, Ingham PW. Contrasting distributions of patched and hedgehog proteins in the Drosophila embryo. Mech Dev 1993; 42:89-96.

(Supplementary information is linked to the online version of the paper on the Cell Research website.)

This work is licensed under the Creative Commons Attribution-NonCommercial-No Derivative Works 3.0 Unported License. To view a copy of this license, visit http:// creativecommons.org/licenses/by-nc-nd/3.0 